\section{- OPEN ACCESS}

\title{
Clinical predictors of rectal lymphogranuloma venereum infection: results from a multicentre case-control study in the UK
}

\author{
S N S Pallawela, ${ }^{1}$ A K Sullivan, ${ }^{1}$ N Macdonald, ${ }^{2}$ P French, ${ }^{3}$ J White, ${ }^{4} \mathrm{G}$ Dean, ${ }^{5}$ \\ A Smith, ${ }^{6} \mathrm{~A} J$ Winter, ${ }^{7} \mathrm{~S}$ Mandalia, ${ }^{2} \mathrm{~S}$ Alexander, ${ }^{8} \mathrm{C}$ sson, $^{8} \mathrm{H} \mathrm{Ward}^{2}$
}

For numbered affiliations see end of article.

\section{Correspondence to} Professor Helen Ward, Department of Infectious Disease Epidemiology, School of Public Health, Imperial College, Norfolk Place, London W2 1PG, UK ; h.ward@imperial.ac.uk.

SNSP and AKS are joint first authors.

Received 19 October 2013 Revised 2 March 2014 Accepted 3 March 2014 Published Online First 31 March 2014

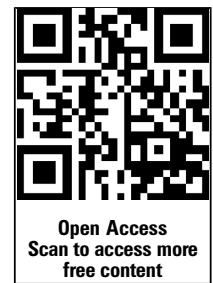

\section{SLinked}

- http://dx.doi.org/10.1136/ sextrans-2013-051404 http://dx.doi.org/10.1136/ sextrans-2013-051386

\section{ABSTRACT}

Objective Since 2003, over 2000 cases of

lymphogranuloma venereum (LGV) have been diagnosed in the UK in men who have sex with men (MSM). Most cases present with proctitis, but there are limited data on how to differentiate clinically between LGV and other pathology. We analysed the clinical presentations of rectal LGV in MSM to identify clinical characteristics predictive of LGV proctitis and produced a clinical prediction model.

Design A prospective multicentre case-control study was conducted at six UK hospitals from 2008 to 2010 Cases of rectal LGV were compared with controls with rectal symptoms but without LGV.

Methods Data from 98 LGV cases and 81 controls were collected from patients and clinicians using computer-assisted self-interviews and clinical report forms. Univariate and multivariate logistic regression was used to compare symptoms and signs. Clinical prediction models for LGV were compared using receiver operating curves

Results Tenesmus, constipation, anal discharge and weight loss were significantly more common in cases than controls. In multivariate analysis, tenesmus and constipation alone were suggestive of LGV (OR 2.98, $95 \% \mathrm{Cl} 0.99$ to 8.98 and $2.87,95 \% \mathrm{Cl} 1.01$ to 8.15 , respectively) and that tenesmus alone or in combination with constipation was a significant predictor of LGV (OR $6.97,95 \% \mathrm{Cl} 2.71$ to 17.92). The best clinical prediction was having one or more of tenesmus, constipation and exudate on proctoscopy, with a sensitivity of $77 \%$ and specificity of $65 \%$.

Conclusions This study indicates that tenesmus alone or in combination with constipation makes a diagnosis of LGV in MSM presenting with rectal symptoms more likely.

\section{INTRODUCTION}

\section{Background}

Since 2003, an outbreak of lymphogranuloma venereum (LGV) has been recognised in Western Europe affecting men who have sex with men $(\mathrm{MSM})^{1}$ with the serovar L2b identified as the causative organism. ${ }^{2}$ By June 2012, 2138 LGV cases had been diagnosed in the UK with $99 \%$ in MSM, the majority with established HIV infection. ${ }^{3}$

In this current outbreak, LGV usually presents as a primary proctitis, sometimes associated with constitutional symptoms. ${ }^{4}$ Anal discharge and proctitis are commonly reported, ${ }^{5}{ }^{6}$ but there are limited data on the specificity of these symptoms and signs, making it difficult to differentiate clinically between LGV and other infections. LGV proctitis may also resemble inflammatory bowel disease in symptoms, endoscopic findings and histology and may result in referral to other specialties resulting in delayed diagnosis and increased risk of complications. $^{7-11}$ Relatively few MSM diagnosed with LGV have presented with the inguinal syndrome ${ }^{12}$ or perianal ulceration, ${ }^{13}$ and in the UK no significant reservoir of asymptomatic or undiagnosed infection has been identified, in contrast to data from the Netherlands. ${ }^{14-16}$ The persistence of LGV in the UK over nearly a decade shows that control efforts have failed to limit ongoing transmission.

\section{Objectives}

We aim to describe in detail the clinical presentation and course of LGV in MSM, and to identify clinical symptoms and signs predictive of LGV compared with other forms of proctitis and to produce a clinical prediction model to support the diagnostic process.

\section{METHODS}

\section{Study design}

A prospective multicentre case-control study. This is part of a wider study (LGV-net) that explored the clinical, epidemiological and microbiological characteristics of LGV. ${ }^{17}$ In the study of risk factors for LGV acquisition, two control groups were used, symptomatic and asymptomatic. However, in the analysis presented here, we are using only the symptomatic controls as we aim to identify the specific symptoms and clinical characteristics distinguishing LGV from other presentations.

\section{Setting}

Subjects were recruited from Genitourinary Medicine, HIV and Sexual Health Clinics, based in six hospitals located in London, Brighton and Glasgow, selected to include those seeing significant numbers of cases of LGV in the UK. National ethics committee approval was granted for the study (07/H0712/156) and individual informed patient consent obtained.

\section{Participants}

Cases were MSM diagnosed with rectal LGV (included rectal asymptomatic and presymptomatic patients) between August 2008 and December 
2010 at one of the participating clinics. Controls were selected (one control per case) by staff involved in the study, from MSM presenting to the same centre during the same week as the case. The inclusion criteria for controls were patients presenting with symptoms of proctitis, anogenital ulceration or inguinal lymphadenopathy who tested negative for LGV and who had reported anogenital sex with a man in the previous 3 months. We aimed to recruit one symptomatic control for each case from the same centre, and seen in the same date period as the case. All controls reported symptoms of proctitis, some also had perianal ulceration. Ten patients initially recruited as symptomatic controls and nine asymptomatic controls who were subsequently diagnosed with LGV were transferred to the LGV case group and further controls recruited. Men who were unable to provide informed consent, who lacked sufficient English or were unable to complete the computer-assisted self-interviews (CASIs) were not eligible.

\section{Investigations}

Cases and controls were investigated using standard clinic protocols, including testing for chlamydia and gonorrhoea from rectum, urethra/urine and pharynx by a variety of NAATs. All samples from cases and controls that tested positive for Chlamydia trachomatis were sent to the relevant Sexually Transmitted Bacterial Reference Laboratory (STBRL) in England or Scotland for LGV testing using an LGV-specific real-time PCR assay. ${ }^{18}$ Proctoscopy was carried out on all those with rectal symptoms unless there were clinical contraindications, such as significant pain or the patient declined. Serological testing for HIV, syphilis and hepatitis C was undertaken according to clinical need.

\section{Data collection and handling}

Patients completed a web-based CASI providing detailed accounts of sociodemographics, risk behaviour and previous medical history. A separate web-based clinical report form (CRF) was completed by the recruiting team, detailing patient symptoms, relevant past medical and sexual history, examination findings and results of investigations. Patients were offered a full sexual health screen and received standard clinical care. Cases were followed up by undergoing a test of cure 4 weeks and 6 months after completion of treatment and re-screened for all sexually transmitted infections (STIs) including HIV, syphilis and hepatitis $\mathrm{C}$ if initially negative. Cases were asked to complete a further CASI at the test of cure follow-up concerning experiences of LGV symptoms and treatment.

Data from the online CASI and the CRF were linked using a unique study number; no personal identifying information was collected. Data were then combined into a standard database format, cleaned and exported to a statistical package for analysis.

\section{Main outcome measure and statistical analysis}

A preliminary descriptive univariate analysis was carried out comparing the clinical presentation and findings for cases and controls. Continuous variables were initially explored using $t$ tests. Missing data were omitted. Key symptoms and signs $(p<0.05)$ predictive of LGV were then analysed further in a multivariable logic regression model. The final multivariable model presented shows significant independent predictors of LGV adjusted for the residual or confounding effects of other variables in the model. Using the results of the univariate and multivariate analysis, we constructed alternative clinical prediction models for LGV in men with rectal symptoms. Symptoms and signs with the largest ORs were entered into different models and receiver operating curves plotted of 1-specificity against sensitivity to identify an algorithm to support the diagnosis of LGV.

\section{RESULTS}

\section{Participants}

In total, 98 rectal LGV cases and 81 symptomatic controls between August 2008 and December 2010. The recruitment rate was $84 \% ; 78 \%$ for cases and $87 \%$ controls.

\section{Descriptive data}

Both cases and controls were mainly men in their 30 s and $40 \mathrm{~s}$, around half of whom were born in the UK, and the majority of whom reported previous STIs (table 1). Cases were significantly more likely than controls to be of white ethnicity (90\% vs $78 \%)$, to be coinfected with HIV (90\% vs 72\%) and to have had one or more previous STIs (99\% vs $84 \%$ ). Controls had a wide range of eventual diagnoses: 44 had one or more STIs (table 2), several patients had coinfections, 30 had no specific diagnosis and 7 had non-STI diagnoses ( 3 haemorrhoids, 2 anal fissures, 1 giardia and 1 microsporidiosis). Ten (10\%) LGV cases and eight (10\%) symptomatic controls gave a history of hepatitis C. Six LGV cases had a recently (within 6 months) positive hepatitis C PCR with no previous history of hepatitis $\mathrm{C}$ detected at the follow-up visit for LGV. Among the nine LGV cases known not to have HIV infection at baseline, one seroconverted for HIV within the 6-month follow-up.

Some cases had other STIs in addition to rectal LGV (table 2). LGV was also detected in the pharynx in one patient who presented with rectal symptoms but no pharyngeal symptoms. However, screening for pharangeal $C$ trachomatis was not routine at most of the participating clinics and was carried out for only 20 cases and 24 controls included in this study.

Although the majority of the cases had rectal symptoms, nine reported no rectal symptoms at the time of their initial positive test for C trachomatis. Of these, four were contacts of LGV and the remainder reported other genital symptoms. One month after completion of treatment, five of the nine reported having developed at least one rectal symptom in the period between testing and treatment, two reported no symptoms throughout and two did not complete the follow-up questionnaire where LGV symptoms were recorded.

Median duration of symptoms was 13 days in cases and 7 days in controls, although eight cases and eight controls reported being symptomatic for over a month before seeking care. The most common symptoms in men with LGV were rectal discharge (66\%), bleeding (61\%) and anal pain $(56 \%)$, followed by tenesmus (33\%) and a change in bowel habit, with around one in three reporting constitutional symptoms (table 3). Tenesmus, constipation, anal discharge and weight loss were significantly more common in cases than controls. A similar proportion of cases and controls also reported genital symptoms (10\% and 15\%, respectively), mostly dysuria and urethral discharge.

Proctoscopy was performed on 81 (83\%) cases and 73 (90\%) controls (table 3) with cases significantly less likely to be reported as 'normal' and more likely to have proctitis, exudate and bleeding, with exudate and rectal bleeding more significantly associated with LGV on univariate analysis.

\section{Multivariable analyses}

In a multivariate model combining these symptoms and signs (adjusting for other variables), tenesmus and constipation alone 
Table 1 Clinic attendance, demographic information and history of sexually transmitted infections for rectal LGV cases compared with symptomatic controls

\begin{tabular}{|c|c|c|c|c|}
\hline Characteristic & $\begin{array}{l}\text { Cases } \\
\mathrm{N}(\%)\end{array}$ & $\begin{array}{l}\text { Controls } \\
\mathrm{N}(\%)\end{array}$ & OR $(95 \% \mathrm{Cl})$ & p Value \\
\hline & $\mathrm{N}=98$ & $\mathrm{~N}=81$ & & \\
\hline \multicolumn{5}{|l|}{ Recruitment setting } \\
\hline HIV clinic & $65(66)$ & $55(68)$ & Ref & $0.757^{*}$ \\
\hline Genitourinary medicine clinic & $27(28)$ & $23(28)$ & $0.99(0.51$ to 1.93$)$ & \\
\hline Othert & $6(6)$ & $3(4)$ & 1.69 (0.40 to 7.08$)$ & \\
\hline \multicolumn{5}{|l|}{ Reason attended clinic } \\
\hline Symptoms & $75(78)$ & $72(89)$ & Ref & $0.075^{*}$ \\
\hline Routine/check up & $12(12)$ & $3(4)$ & $3.84(1.04$ to 14.17$)$ & \\
\hline Other & $9(9)$ & $6(7)$ & 1.44 (0.49 to 4.25$)$ & \\
\hline Age median (IQR) & $39.5(34-46)$ & $37(30-44)$ & $\mathrm{n} / \mathrm{a}$ & $0.087 \S$ \\
\hline Ethnicity —white (UK or other) & $87(90)$ & $63(79)$ & 2.35 (1.01 to 5.47$)$ & 0.048 \\
\hline Born in UK & $54(56)$ & $53(65)$ & $0.66(0.36$ to 1.22$)$ & 0.186 \\
\hline Known HIV-positive & $88(90)$ & $58(72)$ & $3.49(1.55$ to 7.87$)$ & 0.003 \\
\hline Previous hepatitis C & $10(10)$ & $8(10)$ & $1.02(0.38$ to 2.73$)$ & 0.964 \\
\hline Previous gonorrhoea & $67(68)$ & $43(54)$ & 1.86 (1.01 to 3.43$)$ & 0.047 \\
\hline Previous syphilis & $47(48)$ & $31(39)$ & $1.46(0.80$ to 2.65 & 0.219 \\
\hline Previous LGV & $16(16)$ & $8(10)$ & $1.76(0.71$ to 4.34$)$ & 0.223 \\
\hline Previous chlamydia (non-LGV) & $51(52)$ & $30(38)$ & 1.81 (0.99 to 3.30) & 0.054 \\
\hline Any previous sexually transmitted infection & 97 (99) & $67(84)$ & $18.82(2.4$ to 147.3$)$ & 0.005 \\
\hline
\end{tabular}

were suggestive of LGV, with an OR of 2.98 (95\% CI 0.99 to 8.98 ) and 2.87 (95\% CI 1.01 to 8.15 ), respectively. Constipation or tenesmus occurred in approximately half (47\%) of LGV cases in our study. If tenesmus alone, or in combination with constipation, was present, this was highly suggestive that LGV was the cause of the rectal symptoms, with an OR of 6.97 (95\% CI 2.71 to 17.92). These associations were modified slightly if rectal gonorrhoea was added to the model, with tenesmus becoming of borderline significance (aOR 2.82, 95\% CI 0.99, 8.86), constipation becoming more strongly associated (aOR 3.45, 95\% CI 1.19 to 10.04 ) and gonorrhoea itself being an independent predictor of LGV (aOR 4.02, 95\% CI 1.17 to 13.80 ).
We assessed potential clinical prediction models using combinations of the following symptoms and signs: tenesmus, constipation, weight loss, exudate and bleeding on proctoscopy, and a normal proctoscopy. The best balance of sensitivity and specificity was found in an algorithm combining tenesmus, constipation and exudate on proctoscopy; having at least one of these symptoms and signs gives a sensitivity of $77 \%$ and specificity of $65 \%$ (figure 1). We did not include gonorrhoea in the model since this would not be usually known at initial presentation unless suspected on Gram stain on microscopy.

The treatment regimen was recorded for 95 (97\%) of the cases: the majority $(90 / 95,95 \%)$ received the recommended

Table 2 Sexually transmitted infections diagnosed in rectal LGV cases and symptomatic controls

\begin{tabular}{|c|c|c|c|c|}
\hline Characteristic & $\begin{array}{l}\text { Cases } \\
\mathrm{N}(\%)\end{array}$ & $\begin{array}{l}\text { Controls } \\
\mathrm{N}(\%)\end{array}$ & OR $(95 \% \mathrm{Cl})$ & p Value \\
\hline & $\mathrm{N}=98$ & $\mathrm{~N}=81$ & & \\
\hline Rectal chlamydia (non-LGV) & $\mathrm{n} / \mathrm{a}$ & $20(25)$ & $\mathrm{n} / \mathrm{a}$ & \\
\hline Urethral chlamydia & $5(6)$ & $3(4)$ & $1.43(0.33$ to 6.20$)$ & 0.629 \\
\hline Pharyngeal chlamydia* & 4 & 0 & $\mathrm{n} / \mathrm{a}$ & \\
\hline Rectal gonorrhoea & $18(19)$ & $6(8)$ & 2.81 (1.06 to 7.46$)$ & 0.038 \\
\hline Urethral gonorrhoea & $3(3)$ & $2(3)$ & $1.28(0.21$ to 7.85$)$ & 0.791 \\
\hline Pharyngeal gonorrhoea & $2(2)$ & $2(2)$ & 0.89 (0.12 to 6.44$)$ & 0.905 \\
\hline Non-gonococcal urethritis & $7(8)$ & $2(3)$ & 3.09 (0.62 to 15.32$)$ & 0.168 \\
\hline Anogenital herpes & $2(22)$ & $6(8)$ & 0.26 (0.05 to 1.34$)$ & 0.168 \\
\hline Anogenital warts & $8(9)$ & $10(13)$ & $0.64(0.24$ to 1.71$)$ & 0.371 \\
\hline Syphilis (new infection) $\dagger$ & $1(1)$ & $5(7)$ & $0.17(0.02$ to 1.46$)$ & 0.105 \\
\hline
\end{tabular}


Table 3 Presenting symptoms and proctoscopic findings in rectal LGV cases and symptomatic controls

\begin{tabular}{|c|c|c|c|c|}
\hline Characteristic & $\begin{array}{l}\text { Cases } \\
\mathbf{n}(\%)\end{array}$ & $\begin{array}{l}\text { Controls } \\
\text { n (\%) }\end{array}$ & OR $(95 \% \mathrm{Cl})$ & p Value \\
\hline Total & 98 & 81 & & \\
\hline Any rectal symptoms & $89(91)$ & $81(100)$ & $\mathrm{n} / \mathrm{a}$ & \\
\hline Duration: median days (IQR) & $13(5-21)$ & $7(3.5-14.5)$ & $\mathrm{n} / \mathrm{a}$ & $0.146^{*}$ \\
\hline Anal discharge & $65(66)$ & $36(44)$ & 2.46 (1.34 to 4.51$)$ & 0.004 \\
\hline Rectal bleeding & $60(61)$ & $41(51)$ & $1.54(0.85$ to 2.79$)$ & 0.155 \\
\hline Anal pain & $55(56)$ & $36(44)$ & $1.60(0.88$ to 2.89$)$ & 0.121 \\
\hline Tenesmus & $32(33)$ & $6(7)$ & $6.06(2.39$ to 15.40$)$ & $<0.001$ \\
\hline Constipation & $29(30)$ & $8(10)$ & 3.84 (1.64 to 8.96$)$ & 0.002 \\
\hline Loose stools/diarrhoea & $28(29)$ & $20(25)$ & $1.22(0.63$ to 2.38$)$ & 0.560 \\
\hline Any constitutional symptoms & $33(34)$ & $21(26)$ & 1.45 (0.76 to 2.78$)$ & 0.265 \\
\hline Malaise & $19(20)$ & $8(10)$ & 2.19 (0.90 to 5.32$)$ & 0.082 \\
\hline Fever & $16(16)$ & $8(10)$ & $1.78(0.72$ to 4.40$)$ & 0.213 \\
\hline Weight loss & $11(11)$ & $1(1)$ & $10.10(1.28$ to 80.06$)$ & 0.029 \\
\hline Any genital or inguinal symptoms & $11(11)$ & $12(15)$ & $0.73(0.30$ to 1.75$)$ & 0.476 \\
\hline Proctoscopy findings $†$ & $81(91)$ & $73(91)$ & & \\
\hline Normal & $8(10)$ & $16(22)$ & 0.39 (0.16 to 0.97$)$ & 0.044 \\
\hline Proctitis & $52(65)$ & $34(47)$ & 2.08 (1.08 to 3.98$)$ & 0.028 \\
\hline Rectal bleeding & $31(39)$ & $13(18)$ & 2.87 (1.36 to 6.08$)$ & 0.006 \\
\hline Exudate & $47(59)$ & $20(28)$ & 3.70 (1.87 to 7.32$)$ & $<0.001$ \\
\hline Lesion or/ulcer & $13(16)$ & $10(14)$ & $1.22(0.50$ to 3.00$)$ & 0.661 \\
\hline
\end{tabular}

*Wilcoxon rank sum test for medians.

†Proctoscopy was not performed in 17 cases (9 asymptomatic, 1 unable to be performed because of pain, 2 had perianal lesions only, 1 declined, 4 reason not recorded) and 8 controls

( 2 because of pain, 3 perianal lesions, 1 abdominal cramps, 2 reason not recorded).

LGV, lymphogranuloma venereum.

dose of doxycycline (100 mg twice daily for 3 weeks), 71 of whom had this as first line, the remainder once the diagnosis was confirmed.

\section{DISCUSSION}

Tenesmus, constipation, anal discharge and weight loss were significantly more common presenting symptoms in men with LGV compared with controls. In multivariate analysis, constipation and tenesmus alone were suggestive of having LGV; men with tenesmus alone or in combination with constipation were almost seven times more likely to have LGV. The median duration for rectal symptoms for LGV cases was 13 days, ranging from 1 day to a year, similar to that reported elsewhere. ${ }^{19}$ Proctoscopic findings helped differentiate cases and controls, with exudate or bleeding being significant predictors of LGV on univariate analysis.

We developed a simple clinical algorithm to assist in the diagnosis of LGV in men with rectal symptoms: having one or more of tenesmus, constipation and exudate on proctoscopy had a

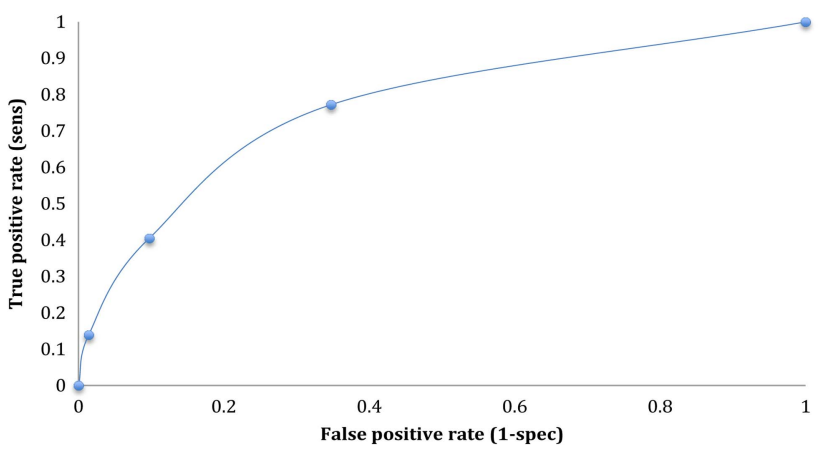

Figure 1 Receiver operator curve for clinical algorithm of tenesmus (1)+constipation (1)+exudate on proctoscopy (1). sensitivity of $77 \%$ and specificity $65 \%$. These parameters are not high enough to be diagnostic, but may improve initial assessment, and presumptive treatment while diagnostic results are awaited. Asymptomatic rectal LGV cases were included in our analyses to reflect clinical practice; however, only 9\% (9/98) of cases had no rectal symptoms at presentation: the majority were 'presymptomatic' and went on to develop symptoms, with only two remaining persistently asymptomatic.

This differs to the $27 \%$ asymptomatic rate observed in the Netherlands, which may reflect LGV testing of all positive rectal chlamydia specimens irrespective of symptoms (Dr Henry de Vries, personal communication). UK practice is for type-specific testing of chlamydia only in symptomatic patients and contacts. $^{20}$

\section{Limitations}

Our study is limited by the high number of clinicians examining patients, and therefore proctoscopic findings and rectal microscopy may not be consistently reported, and not all LGV cases had proctoscopy. Other studies have suggested that proctitis detected by proctoscopic examination together with greater than 10 or 20 poylmorphonuclear leucocytes per high-power field on a rectal smear is suggestive of $\mathrm{LGV}^{56}$

The study is strengthened by the prospective recruitment, multisite setting and systematic data collection using CASI (participants) and online CRFs (clinicians). While these methods do not eliminate variation in data quality, they will have improved the validity of reports on symptoms and signs compared with other studies of LGV that have often been based on retrospective case note review.

\section{Interpretation}

Other studies have reported the association with tenesmus and constipation. $^{21-25}$ Our study is the first to quantify this 
association that has been postulated as resulting from the transmural and perirectal inflammation and oedema caused by $\mathrm{LGV}^{25}$ This study also highlights that patients with LGV present with a wide range of symptoms suggestive of other conditions and are frequently coinfected with other STIs. ${ }^{7}{ }^{26-29}$ Nearly one-fifth of LGV cases were coinfected with rectal gonorrhoea. Most of the cases and controls were coinfected with HIV, in line with other studies in the UK, ${ }^{4}$ and the one case of incident HIV infection reinforces the recommendation for repeat HIV testing and offer of other risk reduction interventions. Importantly, rectal carcinoma should always be considered in those presenting with bleeding and weight loss; however, cases have been reported where LGV has presented with an ulcerating bleeding mass. $^{28} 29$

The majority of patients with LGV were treated with the recommended 3-week course of doxycycline. ${ }^{20}$ Due to the delay in obtaining diagnostic confirmation, treatment should be started presumptively based on clinical symptoms along with gonorrhoea treatment if clinically indicated. Patients confirmed with LGV should be offered a test of cure.

\section{Generalisability}

These findings from a multicentre study should be applicable in areas where LGV has become established among MSM populations. The validity of the clinical predictors is highly dependent on the prevalence of LGV and the proportion of cases that are symptomatic and therefore the findings may not be applicable in settings with a different epidemic pattern for LGV.

\section{CONCLUSION}

LGV is now an established infection among sexually active MSM in the UK, particularly among those with HIV. Clinicians should be aware that MSM presenting with any rectal symptoms should be tested for LGV and treated presumptively, particularly if they have one or more of tenesmus, constipation and exudate on proctoscopy.

\section{Key messages}

- Tenesmus alone, or in combination with constipation, is suggestive of lymphogranuloma venereum (LGV) in men who have sex with men (MSM).

- If LGV is suspected, presumptive treatment with three weeks of doxycycline should be given.

- LGV should be considered in the differential diagnosis of all MSM presenting with rectal symptoms and tests for other STIs should be carried out.

\footnotetext{
Author affiliations

${ }^{1}$ Chelsea and Westminster Hospital NHS Foundation Trust, London, UK ${ }^{2}$ Department of Infectious Disease Epidemiology, School of Public Health, Imperial College London, London, UK

${ }^{3}$ Mortimer Market Centre, Central and North West London NHS Foundation Trust, London, UK

${ }^{4}$ Guy's and St Thomas' NHS Foundation Trust, London, UK

${ }^{5}$ Brighton \& Sussex University Hospitals NHS Trust, Claude Nicol Centre, Brighton, UK

${ }^{6}$ Jefferiss Wing Centre for Sexual Health, Imperial College Healthcare NHS Trust, London, UK

${ }^{7}$ Sandyford Sexual Health Services, Glasgow, UK

${ }^{8}$ Sexually Transmitted Bacterial Reference Laboratory, Public Health England, London, UK
}

\section{Handling editor Jackie A Cassell}

Acknowledgements We thank the patients who contributed to this study and acknowledge staff at participating clinics, including Wendy Hadley, Andrew Benzie, Lydia Hodson, Mathias Chopin, Claire Schepers, Susannah Halley, Daniel Bradshaw, Keerti Gedela, Huw Price, Alex Azwa, Donal Traynor, and Gary Seaton, the Sexually Transmitted Bacterial Reference Laboratory (STBRL) and all laboratory staff at each centre for testing samples in this study.

Collaborators Wendy Hadley, Andrew Benzie, Lydia Hodson, Mathias Chopin, Claire Schepers, Susannah Halley, Daniel Bradshaw, Keerti Gedela, Huw Price, Alex Azwa, Donal Traynor and Gary Seaton.

Contributors SNSP contributed to the recruitment of patients, data collection, analysis of data and produced the first draft and revision of this paper. AKS contributed to the study design, recruitment of patients, data collection and analysis for this paper and drafting and revision of this paper. NM was the overall study coordinator and contributed to the design, established data collection systems, managed the data and carried out preliminary statistical analysis and commented on early and final drafts of the paper. PF, JAW, GD, AS and AJW contributed to the design of the study, oversaw recruitment in their clinics and contributed to the interpretation of findings. All authors contributed to revising and approving the manuscript. SM carried out the analysis of the data. SA carried out LGV diagnostics and related data management. $\mathrm{Cl}$ contributed to the design and management of the study and manages the LGV diagnostic service at STBRL. HW conceived the study and contributed to its design and management, statistical analysis and the drafting and revision of this paper.

Funding This work was funded by a grant from the Medical Research Council G0601699. HW also received funding from the Imperial NIHR Biomedical Research Centre.

\section{Competing interests None.}

Patient consent Obtained.

Ethics approval Central and local approval were obtained by the relevant Research Ethics Committees.

Provenance and peer review Not commissioned; externally peer reviewed.

Open Access This is an Open Access article distributed in accordance with the terms of the Creative Commons Attribution (CC BY 3.0) license, which permits others to distribute, remix, adapt and build upon this work, for commercial use, provided the original work is properly cited. See: http://creativecommons.org/licenses/ by/3.0/

\section{REFERENCES}

1 Nieuwenhuis RF, Ossewaarde JM, Gotz HM, et al. Resurgence of lymphogranuloma venereum in Western European outbreak of Chlamydia Trachomatis serovar L2 proctitis in the Netherlands among men who have sex with men. Clin Infect Dis 2004;39:996-1003.

2 Spaargen J, Schacter J, Moncada J, et al. Slow epidemic of lymphogranuloma venereum L2b strain. Emerg Infect Dis 2005;11:1787-8.

3 Hughes $\mathrm{G}$, Alexander $\mathrm{S}$, Sims I, et al. Lymphogranuloma venereum diagnoses among men who have sex with men in the U.K.: interpreting a cross-sectional study using an epidemic phase-specific framework. Sex Transm Infect 2013;89:542-7.

4 Ward H, Martin I, Macdonald N, et al. Lymphogranuloma venereum in the United Kingdom. Clin Infect Dis 2007;44:26-32.

5 Hamill M, Benn P, Carter C, et al. The clinical manifestations of Anorectal infection with lymphogranuloma venereum (LGV) versus non LGV strains of Chlamydia trachomatis: a case-control study in homosexual men. Int J STD AIDS 2007;18:472-5.

6 Samarawickrama A, Nambiar K, Collins I, et al. Diagnostic utility of rectal microscopy in predicting lymphogranuloma venereum (LGV) in men who have sex with men (MSM): a case control study_Abstract. Sex Transm Inf 2008;84:e1.P78 B-09.

7 Soni S, Srirajaskanthan R, Lucas SB, et al. Lymphogranuloma venereum proctitis masquerading as inflammatory bowel disease in 12 homosexual men. Aliment Pharmacol Ther 2010;32:59-65.

8 Ahdoot A, Kotler DP, Suh JS, et al. Lymphogranuloma venereum in Human Immunodeficiency Virus-Infected Individuals in New York City. J Clin Gastroenterol 2006:40:385-90.

9 Albay D, Mathisen GE. Head and neck manifestations of Lymphogranuloma venereum. Ear Nose Throat I 2008:87:478-80.

10 Piris RI, Saloojee N, Friedlich M. Lymphogranuloma venereum as a cause of rectal strictures. Can J Surg 2007;50:e31-2.

11 Trebing $D$, Brunner $M$, Kröning $Y$, et al. Tumorous extragenital manifestation of lymphogranuloma venereum. J Dtsch DermatolGes 2005;3:445-7.

12 Sethi G, Allason-Jones E, Richens J, et al Lymphogranuloma venereum presenting as genital ulceration and inguinal syndrome in men who have sex with men in London, UK. Sex Transm Infect 2009;85:165-70.

13 Singhrao T, Higham E, French P. Lymphogranuloma venereum presenting as periana ulceration: an emerging clinical presentation?. Sex Transm Infect 2011;87:123-4. 
14 Ward $\mathrm{H}$, Alexander S, Thornton A, et al. The prevalence of lymphogranuloma venereum infection in men who have sex with men: results of a muticentre case finding study. Sex Transm Infect 2009;85:173-5.

15 Saxon CJ, Hughes G, Ison C, et al. Increasing asymptomatic lymphogranuloma venereum infection in the UK: results from a national case finding study. Sex Transm Infect 2013;89:190-1.

16 de Vrieze NH, van Rooijen M, Schim van der Loeff MF, et al. Anorectal and inguinal lymphogranuloma venereum among men who have sex with men in Amsterdam, The Netherlands: trends over time, symptomatology and concurrent infections. Sex Transm Infect 2013;89:548-52.

17 Macdonald N, Sullivan AK, French P, et al. Risk factors for rectal lymphogranuloma venereum in gay men: results of a multicentre case-control study in the UK. Sex Transm Infect 2014;90:262-8.

18 Morré SA, Spaargaren J, Fennema JSA, et al. Real-time polymerase chain reaction to diagnose lymphogranuloma venereum. Emerg Infect Dis 2005;11:1311-12.

19 Vargas-Leguas H, Garcia de Olalla P, Arando M, et al. Lymphogranuloma venereum: a hidden emerging problem, Barcelona, 2011. Euro Surveill 2012;17:pii20057.

20 White J, O'Farrell N, Daniels D. British Association for Sexual Health and HIV 2013 UK National Guideline for the management of lymphogranuloma vebereum: Clinical Effectiveness Group of the British Association for Sexual Health and HIV (CEG/ BASHH) Guideline development group. Int J STD AIDS 2013;24:593-601.
21 Kamarashev J, Riess CE, Mosiman J, et al. Lymphogranuloma venereum in Zurich, Switzerland: Chlamydia trachomatis serovar L2 proctitis among men who have sex with men. Swiss Med Wkly 2010;140:209-12.

22 Bauwens JE, Lampe MF, Suchland RJ, et al. Infection with Chlamydia trachomatis lymphogranuloma venereum serovar L1 in homosexual men with proctitis: molecular analysis of an unusual case cluster. Clin Infect Dis 1995;20:576-81.

23 Götz HM, Ossewaarde JM, Nieuwenhuis RF, et al. A cluster of lymphogranuloma venereum among homosexual men in Rotterdam with implications for other countries in Western Europe. Ned TijdschrGeneeskd 2004;28:441-2.

24 Hamlyn E, Taylor C. Sexually transmitted proctitis. Postgrad Med J 2006;82:733-6.

25 White J. Manifestations and management of lymphogranuloma venereum. Curr Opin Infect Dis 2009;22:57-66.

26 Song $\mathrm{SH}$, Jang I, Kim BS, et al. A case of primary syphilis in the rectum. J Korean Med Sci 2005;20:886-7.

27 Bassi O, Cosa G, Colavolpe A, et al. Primary syphilis of the rectum- Endoscopic and clinical features, Report of a case. Dis Colon Rectum 1991;34:1024-6.

28 Mistrangelo M, Conte ID, Gregori G, et al. Rectal lymphogranuloma venereum. Colorectal Dis 2012;14:e792-3.

29 Taylor G, Dasari BVM, McKie L, et al. Lymphogranuloma venereum(LGV) proctitis mimicking rectal cancer. Colorectal Dis 2011;13:e63-4. 